\title{
Vorbereitung und Schwerpunkte der Schweiz zu den Traktanden der UNCED
}

Thomas Litscher

\section{(2) OpenEdition}

1 Journals

\section{Electronic version}

URL: http://journals.openedition.org/sjep/1379

DOI: $10.4000 /$ sjep. 1379

ISSN: 1663-9677

\section{Publisher}

Institut de hautes études internationales et du développement

\section{Printed version}

Date of publication: 1 janvier 1992

Number of pages: 183-187

ISSN: $1660-5926$

\section{Electronic reference}

Thomas Litscher, "Vorbereitung und Schwerpunkte der Schweiz zu den Traktanden der UNCED »,

Schweizerisches Jahrbuch für Entwicklungspolitik [Online], 11 | 1992, Online erschienen am: 19 Mai 2013, abgerufen am 08 September 2020. URL : http://journals.openedition.org/sjep/1379 ; DOI : https:// doi.org/10.4000/sjep.1379 


\title{
Vorbereitung und Schwerpunkte der Schweiz zu den Traktanden der UNCED
}

\author{
Thomas Litscher
}

\section{Der Stellenwert von UNCED für die Schweiz}

Die zwei Themen von UNCED - Umwelt und Entwicklung - sind beide von existenzieller Bedeutung. Auch sind die normative Kraft und die Relevanz der von der Konferenz zu erwartenden Ergebnisse erheblich. Dazu gehören: Internationale Abkommen zur Bewältigung der drohenden Klimaveränderung und zur Erhaltung der Artenvielfalt, ein umfassendes Aktionsprogramm - die sogenannte "Agenda 21 " - mit dem den ökologischen und entwicklungspolitischen Herausforderungen im Uebergang ins 21. Jahrhundert begegnet werden soll, eine Charta betreffend die Rechte und Pflichten von Individuen und Staaten im Bereich Umwelt/Entwicklung, sowie eine institutionelle Neuordnung im System der Vereinten Nationen, damit dieses den anstehenden Aufgaben besser gewachsen ist. Ebenso hoch ist der politische Gehalt des UNCED-Prozesses. Es geht um zusätzliche, bedeutende Ressourcentransfers von den Industriestaaten in die Ent-wicklungsländer, um Fragen nachhaltiger Entwicklung, Souveränitätsfragen, Eigentumsrechte, die internationale Wirtschaftsordnung etc. Die Konferenz könnte damit zu einem Meilenstein werden auf dem Weg zur Trendwende weg von der fortschreitenden Umweltzerstörung hin zu einer sozial verträglichen, dauerhaften Entwicklung. Entsprechend misst ihr die Schweiz einen hohen Stellenwert zu.

\section{Die organisatorischen Vorkehren für die Mitwirkung der Schweiz}

Aufgrund der grossen Tragweite von UNCED ist bereits im Vorbereitungsprozess eine aktive schweizerische Mitarbeit und Interessenwahrung nötig. Die Vielzahl und Breite der behandelten Themen und beteiligten Institutionen (UN- Generalversammlung, Prep Com, UNEP, WMO, FAO, UNDP, Weltbank u.a.) stellt hohe Ansprüche an die verwaltungsinterne Koordination, damit in den verschiedenen Gremien eine wirkungsvolle und kohärente Politik verfolgt werden kann. Dem departementsübergreifenden Charakter von UNCED entsprechend hat der Bundesrat das bestehende "Interdepartementale Komitee für Entwicklungszu- 
sammenarbeit und humanitäre Hilfe" mit der Gesamtführung der schweizerischen Mitwirkung an UNCED betraut. Für jeden der sieben für die Schweiz prioritären Bereiche wurde eine departementsübergreifende Arbeitsgruppe eingesetzt, deren Arbeiten von einer Koordinationsgruppe laufend koordiniert werden. Beteiligt sind über zwanzig Bundesämter. Zur inhaltlichen und organisatorischen Stărkung dieser Struktur teilte ihr der Bundesrat befristet für die Dauer des Vorbereitungsprozesses sechs zusătzliche Personaleinheiten zu.

\section{Die Schwerpunkte der Schweiz}

Aus der Vielzahl der UNCED-Themen bestimmte der Bundesrat deren sieben, die prioritär und intensiver zu bearbeiten sind. Diese lassen sich knapp wie folgt charakterisieren:

Nord-Südbelange: Eine grosse Zahl von Themen - von der Armut über das Bevölkenungswachstum bis hin zur internationalen Wirtschafts- und Sozialordnung - steht unter diesem Oberbegriff auf der Tagesordnung. Für die Entwicklungsländer zentral sind allerdings ihre Fordenungen nach zusätzlichen Finanztransfers und erleichtertem Zugang zu Technologien. Die Schweiz unterstützt den Grundsatz, dass für die Beteiligung der Entwicklungsländer an den internationalen Anstrengungen zur Bewältigung der globalen Umweltprobleme zusätzliche Finanzmittel bereitzustellen sind, die die Entwicklungshilfeleistungen ergänzen. Sie setzt sich für einen möglichst zentralen Finanzierungsmechanismus und eine angemessene Lastenteilung ein. Auch die Notwendigkeit eines vermehrten Technologietransfers wird bejaht und diesbezügliche Arbeiten werden unterstützt, unter der Voraussetzung, dass entsprechende Mechanismen schweizerischen ordnungs- und rechtspolitischen Grundsätzen nicht zuwiderlaufen.

- Klimaverănderung: Die Klimaproblematik ist eine der ganz grossen Herausforderungen der Gegenwart. Ohne Massnahmen zur Beschränkung der Trebhausgasemissionen erwarten Wissenschafter eine Erwärmung der Erdoberfläche von zwischen 2 und $5 \mathrm{Grad}$ im Verlauf des nächsten Jahrhunderts. Die Risiken eines solchen Temperaturanstiegs für die Oekosysteme sind enorm (Anstieg des Meeresspiegels, vermehrte und intensivere Unwetter und Stürme, Verschärfung der Trockenheit in ariden und semi-ariden Gebieten usw.) Am unmittelbarsten betroffen von den Auswirkungen dürtten viele Länder der dritten Welt sein. Die Unterzeichnung einer Klimakonvention anlässlich von UNCED wäre damit sicher ein Hauptereignis. Die Schweiz zählt sich mit dem erklärten Regienungsziel, ihre CO2-Emissionen bis ins Jahr 2000 auf dem Niveau von 1990 zu stabilisieren, zu der Gruppe der fortschrittlicheren Länder bezüglich der zu ergreifenden Massnahmen. Sie ist an einer möglichst substanziellen und verpflichtenden Konvention interessiert und hat sich entsprechend stark in den Vorbereitungen der Verhandlungen (z.B. als Gastgeber 
der 2. Weltklimakonferenz vom Herbst 1990 in Genf), wie auch in den Verhandlungen selbst engagiert.

Artenvielfalt: Die Herausfordenung bezüglich der Erhaltung der Biodiversität ist wohl derjenigen der Klimaproblematik ebenbürtig. Die Wissenschaft geht davon aus, dass die Erde durch die Übernutzung, Überlastung, Vergiftung und letztlich Vernichtung von Lebensräumen (insbesondere auch der Tropenwălder) jăhrlich zwischen $250^{\prime} 000$ und $500^{\prime} 000$ Arten verliert. Die Auswirkungen eines derartigen Verlustes an genetischem Material und an Artenvielfalt sind enorm. Abgesehen von einer Verarmung der Natur, einer Störung des okologischen Gleich-gewichts und einer Verringerung der Anpassungsfähigkeit der Oekosysteme ist die Artenvielfalt für Medizin, Chemie und Landwirtschaft von enormem Wert. Die Verabschiedung einer internationalen Konvention zum Schutz der Artenvielfalt anlässlich UNCED wäre damit zweifellos ein weiteres Hauptelement. Die Schweiz unterstützt auch hier die Erarbeitung eines möglichst griffigen, weitgehenden Rechtsinstrumentes.

- Entwaldung: Die Rodung grosser Waldgebiete geht nach jüngsten Schätzungen der FAO weit schneller voran, als noch vor kurzem angenommen. Neueste Untersuchungen gehen davon aus, dass weltweit jährlich ca. 17 Millionen Hektaren Wald abgeholzt werden, eine Fläche, die mehr als viermal derjenigen der Schweiz entspricht. Oekologisch sind die Wälder vor allem als Hort der Artenvielfalt und als klimatisches Regelwerk unschätzbar. Gemäss einer bereits langjährigen Politik des Bundesrates zur Erhaltung der Wălder, insbesondere auch der Tropenwälder, gehört auch dieses Thema zu den Prioritäten. Die Schweiz setzt sich insbesondere für die Erarbeitung von substantiellen Forst-Protokollen unter den Konventionen zu Klima und Artenvielfalt ein. Sie unterstützt gegebenenfalls aber auch die Ausarbeitung einer eigenen Forstkonvention oder von Grundsätzen für eine solche Konvention als nötige Erweiterung und Ergänzung der erwähnten Protokolle.

- Abfall, giftige chemische Substanzen: Die Abfallberge nehmen laufend zu, und die Entsorgung von Sondermüll wird immer schwieriger. Die Schweiz hat sich in diesem Bereich als Initiantin der Basler Konvention über die grenzüberschreitende Verbringung gefährlicher Abfälle und deren Entsorgung bereits seit längerem stark eingesetzt. Es ist daher nur konsequent, dass sie sich in dieser Thematik weiter besonders engagiert. Ebenso unterstützt sie die Arbeiten im Bereich der gefährlichen chemischen Stoffe zur Stärkung des bestehenden Aufsichtsdispositivs.

- Instlitutionelles und Rechtliches: Die bestehenden UN- Institutionen im Umwelt- und Entwicklungsbereich haben alle bereits ein stattliches Alter erreicht. Obwohl sie sich dem Wandel der Problematik entsprechend fortentwickelt haben, verlangt die Dynamik und Tragweite der Umweltproblematik und die zunehmende Erkenntnis ihres inneren Zusammenhanges mit grundlegenden 
Entwicklungsfragen nach einer Überprüfung der Angemessenheit der institutionellen Strukturen und Mittel im UN- System. Das allgemeine schweizerische Interesse an diesen Fragen wird noch verstärkt durch die spezifische Rolle Genfs als Zentrum internationaler Organisationen. Bei den rechtlichen Fragen geht es um die Ausarbeitung einer Charta betreffend Umwelt und Entwicklung, um die Verstärkung der bestehenden Umweltrechtsinstrumente und eventuell um die Entwicklung eines Streitverhinderungs- und -schlichtungsmechanismus im Umweltbereich. Dass die Schweiz, die traditionell der Weiterentwicklung des Völkerrechts einen grossen Stellenwert beimisst, auch diese Arbeiten besonders aufmerksam verfolgt, liegt auf der Hand.

- Nationaler Bericht: Im Rahmen von UNCED ist jeder Staat aufgefordert, einen nationalen Bericht über Umwelt und Entwicklung zu erarbeiten. Entsprechend hat auch die Schweiz diese Arbeit an die Hand genommen. Der Bericht wird auch zur Sensibilisierung für die Thematik und zum Einbezug einer breiteren Oeffentlichkeit in den Vorbereitungsprozess für UNCED dienen.

Selbstverständlich verfolgt die Schweiz auch in den weniger prioritären Themen die Arbeiten des Vorbereitungsprozesses von UNCED. Die dargestellten Prioritäten sind denn auch nicht als festgegeben zu verstehen. Sie könnten entsprechend den Entwicklungen der Arbeiten des Vorbereitungsausschusses von UNCED angepasst werden.

\section{Zusammenfassung}

Bezüglich Entwicklungsländer und Umwelt ist in der jüngeren Vergangenheit das Bewusstsein über zwei Tendenzen stark gewachsen: zum einen die alarmierende Degradation der Umwelt in den Entwicklungsländern selbst und zum anderen deren schnell wachsender Beitrag an die globalen Umweltprobleme. Der Bund trägt dieser Einsicht Rechnung. Er hat Umweltaspekte konsequent in seine Entwicklungszusammenarbeit integriert. Für den schweizerischen Beitrag zur Beteiligung der Entwicklungsländer an den internationalen Anstrengungen zur Bewältigung der globalen Umweltprobleme wurde mit dem Rahmenkredit von 300 Millionen Franken ein neues Instrument geschaffen. Der Vorbereitungsprozess von UNCED bietet der Schweiz ein geeignetes Gefäss, sich für ihre Anliegen im Bereich Umwelt/Entwicklung zu engagieren. 


\section{Literaturverzeichnis}

Schweiz. Bundesrat - Botschaft im Rahmen der 700-Jahrfeier der Eidgenossenschaft über zwei neue Rahmenkredite zur Finanzierung von Entschuldungsmassnahmen zugunsten ärmerer Entwicklungsländer und Umweltprogrammen und projekten von globaler Bedeutung in Entwicklungsländem vom 30. Januar 1991 Schweiz. Bundesrat - Botschaft über die Weiterführung der technischen Zusammenarbeit und der Finanzhilfe zugunsten von Entwicklungsländern vom 21. Februar 1990

Geografisches Institut der Universität Bern - Umweltprobleme und Entwicklungszusammenarbeit, Oktober 1986

Flury Manuel - diverse DEH-interne Notizen 